\title{
Mechanisms of Ligand-Protein Interaction in Sec-14-like Transporters Investigated by Computer Simulations
}

\author{
Rachel E. Helblinga , Christos Lamprakis ${ }^{a}$, Walter Aeschimanna, Cristin S. Bolze ${ }^{a}$, Achim Stocker ${ }^{\star a}$, \\ and Michele Cascella*b
}

\begin{abstract}
We review our recent work on protein-ligand interactions in vitamin transporters of the Sec-14-like protein. Our studies focused on the cellular-retinaldehyde binding protein (CRALBP) and the $\alpha$-tocopherol transfer protein ( $\alpha$-TTP). CRALBP is responsible for mobilisation and photo-protection of short-chain cis-retinoids in the dim-light visual cycle or rod photoreceptors. $\alpha$-TTP is a key protein responsible for selection and retention of RRR- $\alpha$-tocopherol, the most active isoform of vitamin $E$ in superior animals. Our simulation studies evidence how subtle chemical variations in the substrate can lead to significant distortion in the structure of the complex, and how these changes can either lead to new protein function, or be used to model engineered protein variants with tailored properties. Finally, we show how integration of computational and experimental results can contribute in synergy to the understanding of fundamental processes at the biomolecular scale.
\end{abstract}

Keywords: Molecular Dynamics · QM/MM · Metadynamics · Retinal · Tocopherol

\section{Introduction}

Sec-14-like proteins are a class of transporters involved in solubilisation and trafficking of lipophilic molecules among different organelle compartments in the cell and from/to the plasma membrane. The prototypic protein, giving the name to the family, is $\mathrm{Sec} 14 \mathrm{p}$, first identified in Saccharomyces cerevisiae, a protein promoting inter-membrane phosphatidylinositol/phosphatidylcholine exchange in the Golgi apparatus. ${ }^{[1,2]}$

Altogether, there are over 500 proteins harbouring a Sec14 domain in the NCBI database. ${ }^{[3]}$ The family has evolved to fulfil specialised transport functions or more complex tasks at the interface between lipid transport and organelle physiology. Known ligands for Sec14 domains include a wide range of phospholipids, lipids, and hydrophobic vitamins.

\footnotetext{
${ }^{\star}$ Correspondence: Prof. M. Cascella ${ }^{\mathrm{b}}$ Tel.: +4722855420

E-mail: michele.cascella@kjemi.uio.no Prof. Dr. A. Stocker ${ }^{\mathrm{a}}$

Tel.: +41316314315

E-mail: achim.stocker@dcb.unibe.ch

Departement für Chemie und Biochemie

Universität Bern

Freiestrasse 3

$\mathrm{CH}-3012$ Bern

bepartment of Chemistry and Centre for Theoretical

and Computational Chemistry (CTCC)

University of Oslo

PO BOX 1033 Blindern

0315 Oslo, Norway
}

The Sec14 domain in mammalian proteins can also take the name of CRALTRIO, after the cellular retinaldehyde binding protein (CRALBP) and the TRIO guanine exchange factor, the first mammalian protein of the family to be characterised. [4] Globular proteins of this family present a two-lobed structure formed by $\sim 285$ amino acids.

The carboxy-terminal lobe contains the proper CRAL-TRIO motif, and it is structured as an $\alpha / \beta$ domain. The interface between the $\alpha$-helices and the five structurally conserved parallel $\beta$-strands form a large hydrophobic cavity, accommodating the lipophilic substrates. In all proteins, a highly conserved helix acts as a mobile

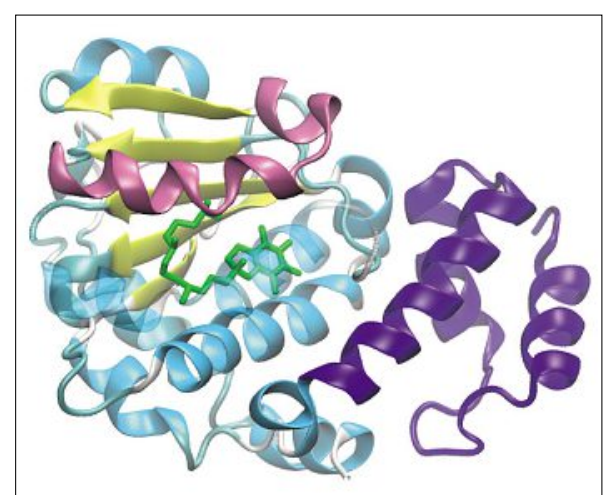

Fig. 1. General fold of Sec-14-like proteins. The $\mathrm{N}$-terminal domain is coloured in purple, the $\alpha$-helices belonging to the proper CRAL-TRIO domain are coloured in cyan, the mobile lid in pink, and the $\beta$-strand at the binding cavity in yellow. The binding cavity is also evidenced by one model ligand (green licorice). gate, opening or closing the access to the binding pocket from the exterior (Fig. 1).

The gate presents two conformations: a closed one, when the ligand is bound inside the binding pocket, and an open one for the apo-protein, as highlighted by the two crystal structures resolved for $\alpha$-TTP (PDB codes: 1OIZ, 1OIP). [5]

The amino-terminal lobe contains four antiparallel $\alpha$-helices, and its function is associated with recognition and binding to the different cellular compartments.

The characterisation of the molecular properties of Sec-14-like proteins, and especially their interaction with substrates, may not be straightforward from the experimental point of view. Difficulties come from the high hydrophobicity of the substrates in the presence of hydrophilic globular proteins. The use of surfactants for solubilisation of the substrates unavoidably leads to biphase systems, which consequently may affect the kinetics of substrate binding into the respective proteins. As a consequence, determination of dissociation constants, relative binding affinities and sometimes even the natural substrate may be particularly difficult. ${ }^{[6,7]}$

Computer simulations and molecular dynamics studies offer a valid method of investigation that can complement information not easily accessible from in vitro studies. In the past few years, we have been focusing our attention on two proteins of the Sec-14-like family, namely: cellular-retinaldehyde binding protein (CRALBP), $\alpha$-tocopherol transfer protein $(\alpha-T T P) .{ }^{[7-10]}$ In more recent times, we 
have also extended our studies to supernatant protein factor (SPF). ${ }^{[11]}$

Here, we briefly review some of our most recent results on our studies on CRALBP and $\alpha$-TTP, adding some additional insights from simulations on the structure/function relationship in these proteins.

\section{CRALBP}

CRALBP is a $36 \mathrm{kDa}$ soluble protein belonging to the Sec14-like protein family. ${ }^{[6,12]}$ It was discovered in 1977 in bovine retina cytoplasm through its ability to bind exogenous radioactive 11-cis-retinoids. ${ }^{[13,14]}$ CRALBP is an essential transporter involved in the recycling of all-trans-retinal to 11-cis-retinal, playing a hinge role in the retinoid visual cycle in the retina pigment epithelium. ${ }^{[15-17]}$ CRALBP binds and transports 11-cis-retinol and assists the oxidation of the retinoids to 11-cis-retinal.[17-19] CRALBP is also expressed in the Müller glial cells, where it assists the esterification of 11-cis-retinol.[20] Expression of CRALBP in the brain, as well as in other tissues like the cornea, pineal gland, ciliary body and embryo hints that it may be involved in other physiological functions than image forming signalling. ${ }^{[21,22]}$

CRALBP protects cis-retinoids from premature isomerization and from enzymatic back transformation into all-trans retinal, by tight steric, non-covalent binding of its substrate. ${ }^{[23-25]}$ The binding pocket of CRALBP is a highly hydrophobic horseshoe-shaped cavity, where the only hydrophilic interactions with the natural 11-cis-retinol/al substrates occur by H-bonding of the alcohol/aldehyde group of the substrate with the side-chains of E202 and Y180 (Fig. 2).[23]

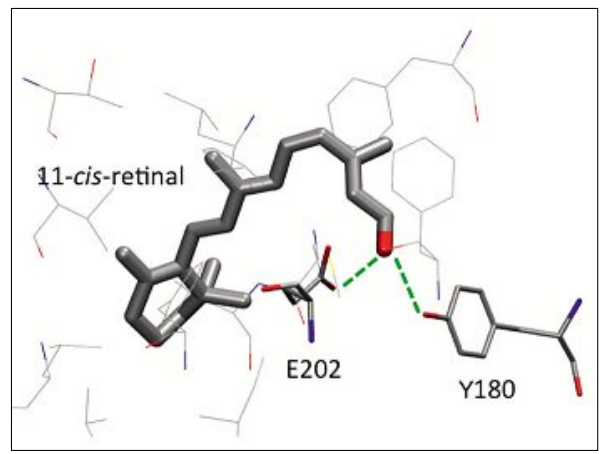

Fig. 2. Binding mode of 11-cis-retinal in CRALBP. Crystallographic image from PDB code: $3 \mathrm{HX} 3 .^{[23]}$ Stabilisation of the aldehyde head by $\mathrm{H}$-bonding with residues $\mathrm{E} 232$ and $\mathrm{Y} 180$ is highlighted by dashed green lines.

Substitution mutations of the CRALBP coding gene $R L B P 1$ are known to cause severe genetic diseases, like autosomal recessive retinitis pigmentosa, night blindness, delayed dark adaptation phenotype, retinitis punctata and Bothnia dystrophy.[26-28] The missense mutationassociated to the latter disease consists in an substitution of an arginine with a tryptophan residue at position 234 , located in a basic patch at the protein surface, ${ }^{[23,29]}$ putatively responsible for establishing initial membrane contacts. ${ }^{[12,30]}$

Experimental data show a smaller cavity for the ligand in the R234W mutant than in the wild-type protein. The contraction of the volume in the binding cavity produces tighter binding of 11-cis-retinal: dissociation constants change from $\mathrm{K}_{\mathrm{d}} \sim 21.0$ $\mathrm{nM}$ in native CRALBP to $\sim 10.3 \mathrm{nM}$ in the R234W mutant.[18] This may affect the ability of CRALBP to release 11-cis-retinal at the end of the transport cycle. ${ }^{[23,30]}$

Both photochemical and UV/vis spectroscopic data demonstrated that CRALBP is able to bind 9-cis-retinoids. ${ }^{[13,18,25]}$ In particular, J. W. Crabb and co-workers determined equilibrium dissociation constants of $\mathrm{K}_{\mathrm{d}} \sim 53.3$ and $\mathrm{K}_{\mathrm{d}} \sim 24.3 \mathrm{nM}$ for native CRALBP and R234W respectively, thus finding only slight less efficiency than the native 11-cis substrates. ${ }^{[18]}$ Such findings connect to other independent studies evidencing that the proteins of the visual cycle are promiscuous and can produce and function with different retinoid isomers. [9]

In the past years, we have been working on the characterisation of 9-cis-retinal binding to both native CRALBP and its R234W mutant. As a starting point, the structures of both CRALBP and R234W in complex with 9-cis-retinal were resolved by X-ray diffraction. ${ }^{[8]}$ The weaker binding of the native form did not allow proper resolution of the structure of the ligand, which is instead visible in R234W. Similar resolution issues were found in earlier crystals of the 11-cis-retinal:CRALBP complex, ${ }^{[23]}$ confirming that R234W binds more tightly to its ligands.

The reconstruction of the electronic density map for the ligand (at $1.9 \AA$ resolution) evidenced a distorted configuration of the molecule, not compatible with any cis- or trans-poly-ene chain. The crystal structures also showed a different binding mode of the aldehyde head, which does not interact with Glu202, but connects to it by a H-bond network of two buried water molecules.

In order to address the molecular details of ligand binding in CRALBP, we performed a set of independent molecular dynamics (MD) simulations on different complexes, namely: i) 11-cis-retinal:CRALBP; ii) 11-cis-retinal:R234W; iii) 9-cis-retinal:CRALBP; iv) 9-cis-retinal:R234W; v) 9-cis-retinol:CRALBP; vi) 9,13-dicis-retinal:CRALBP. ${ }^{[10]}$
Moreover, we performed different structural optimizations of the crystal structure by hybrid QM/MM simulations ${ }^{[31-33]}$ in order to determine the chemical nature of the molecular moieties detected in the $\mathrm{X}$-ray experiment (the technical details are found in the original publication). ${ }^{[8]}$

MD studies evidenced that ligands in the binding pocket of CRALBP are more mobile than in that of R234W. In particular, the 12s-bond of 11-cis-retinoid is forced into a cis-conformation, while in CRALBP it is mostly in a trans-conformation, similar to what was originally observed in free retinal. ${ }^{[34]}$

More importantly, our studies revealed that the water present in the binding cavity of R234W can approach $\mathrm{C} 12$ of 9-cis-retinal to distances shorter than 2 A.. ${ }^{[10]}$ Parallel QM/MM studies could prove that the chemical species detected in the crystal structures was a damaged isoform of 9-cis-retinal produced by $\mathrm{H}$ atom attachment to C12. In fact, the computational model has a RMSD from the best fit from the experimental electron density map of $0.11 \AA$ (Fig. 3). All other chemical structures tested have a significantly larger deviation, especially in the distorted region. ${ }^{[8]}$

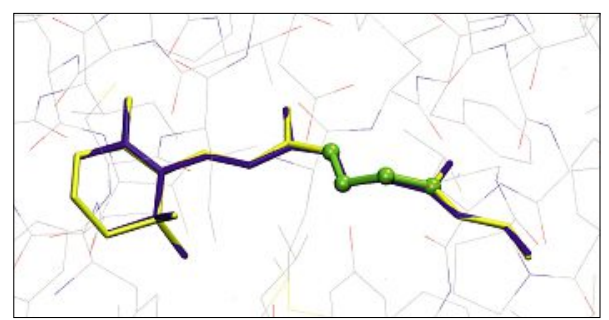

Fig. 3. Superposition of the crystallographic image of 9-cis-retinal bound to the R234W mutant of CRALBP (blue licorice) and the QM/MM model of 9-cis-retinal in the same protein cavity after $\mathrm{H}$ attachment at $\mathrm{C} 12$ (yellow licorice). The distorted $\mathrm{C} 10-\mathrm{C} 11-\mathrm{C} 12-\mathrm{C} 13$ dihedral angle is evidenced in lime CPK. ${ }^{[8]}$

The detected radical damage is most probably formed upon capture of a solvated electron produced by X-ray scattering, which triggers the transfer of a proton from E202 through the buried waters.

Both the nature of the radical-damaged structure, and the hydration patterns found in the computational models of the undamaged complexes suggest that protons can be transiently transferred to retinal at different positions. In fact, incubation of 9-cis-retinal:CRALBP complexes at body temperature, followed by elution and chromatographic analysis of the substrates, revealed slow formation of a new chemical species, which was characterized as 9,13-dicis-retinal by subsequent ${ }^{1} \mathrm{H}-\mathrm{NMR}$ and UV/vis spectroscopy. ${ }^{[8]}$

Strikingly, the reaction appears to be irreversible. In fact, slow quantitative trans- 
formation of the substrate into the product occurs in roughly one day. Even more puzzling, the reaction in $\mathrm{R} 234 \mathrm{~W}$ is faster, and leads to thermodynamic equilibration between 9-cis- and 9,13-dicis-retinal. ${ }^{[8]}$

Our MD simulations of the 9,13-dicis-retinal:CRALBP complex revealed that water molecules are able to exchange with the exterior, and that a different hydration pattern forms in the binding pocket. ${ }^{[10]}$ Experimental kinetic isotope effect measurements evidenced that the proton shuttling required for the formation of the reactive enol species is the key rate-limiting step. ${ }^{[8]}$ Taken together, these data suggest that the irreversibility of the process is induced by the change in the H-bond network in the binding cavity after production of 9,13-dicis-retinal, which reduces the efficiency of the proton shuttling mechanism. This hypothesis needs to be verified by future computational studies.

\section{3. $\alpha$-TTP}

$\alpha$-TTP is a $32 \mathrm{kDa}$ cytosolic protein $^{[35,36]}$ isolated in both rats and humans, responsible for regulation of vitamin $\mathrm{E}$ in hepatocytes. ${ }^{[37]} \alpha$-TTP is mostly expressed in the liver, but it is also present in the placenta, in the brain, ${ }^{[38]}$ as well as in Müller glial cells of the retina. ${ }^{[39]}$

In the liver, the main role of $\alpha$-TTP is to identify, select and extract the RRR$\alpha$-tocopherol isoform of vitamin E ( $\alpha$ Tol hereafter) from maturing endosomal compartments. Correct expression of $\alpha$-TTP is essential to the health of the organism, as its poor expression or mutation is directly associated to occurrence of ataxia with vitamin E deficiency (AVED) disease.

As shown in Fig. 4, there are two main cellular steps involving $\alpha$-TTP: the first, occurring at the endosome, where the protein must recognise and select $\alpha$ Tol, and the second, at the plasma membrane, where $\alpha$ Tol must be released into the blood stream. Both passages are still debated in the literature. In both cases, $\alpha$-TTP must recognise and bind to the membrane. Site-directed mutagenesis experiments on $\alpha$-TTP hydrophobic surface residues (especially F165 and F169) showed a decrease of both protein adsorption on the lipid bilayer and the rate of inter-membrane ligand transfer. ${ }^{[40]}$

A decade ago, the first crystallographic structures of the $\alpha$ Tol: $\alpha$-TTP complex were resolved. ${ }^{[5,41]}$ Based on the original crystallographic data, it was proposed that the selectivity came from optimization of van der Waals contacts between $\alpha$ Tol and the surrounding protein environment. ${ }^{5,41-44]}$ Nonetheless, a pure enthalpic effect based on loss of hydrophobic contacts could not easily explain the extent of

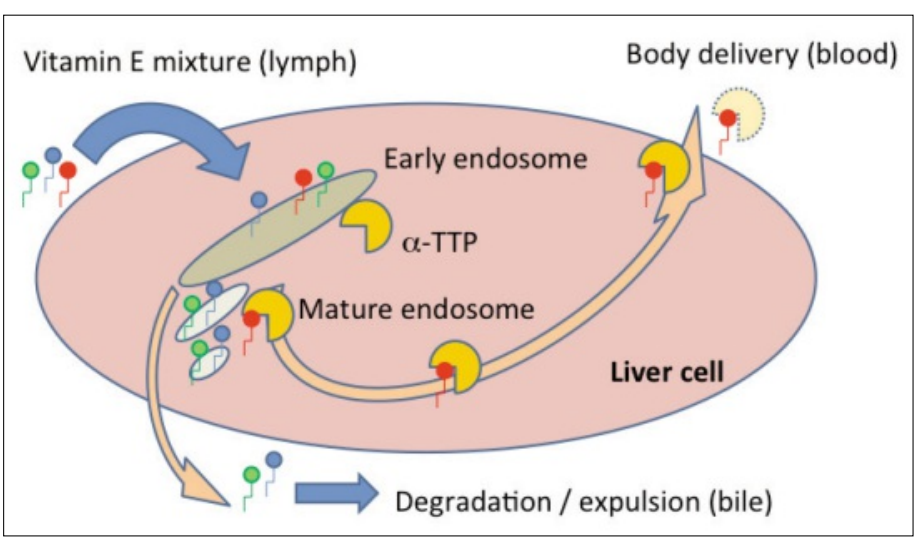

Fig. 4. Mechanism of selection of $\alpha$-tocopherol (red drawing) in liver cells.

the effect on binding affinity, and also the variation of affinity between isoforms containing the same number of methyl groups at the chromanol ring.

In more recent times, we tackled the problem of ligand selectivity in $\alpha$-TTP using a combined computational/experimental approach. ${ }^{[7]}$ We concentrated on the difference of binding between $\alpha$ Tol and $\gamma$-tocopherol ( $\gamma$ Tol) (Fig. 5), which is an isoform of vitamin $\mathrm{E}$ highly bioavailable in several dietary regimes, potentially leading to vitamin E deficiencies in the exposed population.

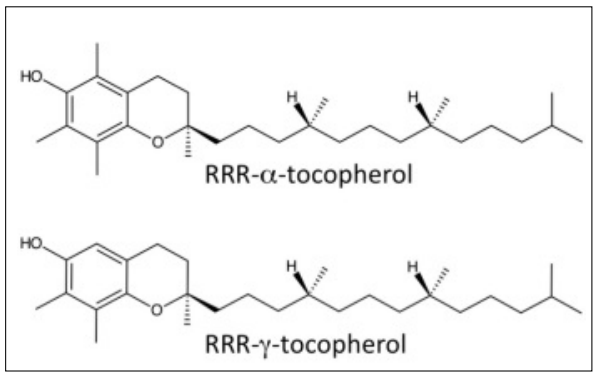

Fig. 5. Chemical structures of $\alpha$ Tol and $\gamma$ Tol.
Computer simulations of the $\alpha$ Tol: $\alpha$ TTP and $\gamma$ Tol: $\alpha$-TTP complexes revealed that the minimal variation in the chemical structure of the ligands has a large impact in the equilibrium structure of the complex. In particular, the chromanol ring of $\gamma$ Tol is not stable in the binding position of $\alpha \mathrm{Tol}$, producing also a rearrangement of the hydrophobic isoprenoid tail with respect to the binding position of $\alpha$ Tol (Fig. 6). ${ }^{[7]}$

Our simulations revealed that the chromanol ring of $\gamma$ Tol is in closer contact to the methyl side-chain of A156 than $\alpha$ Tol. Therefore, our model suggested that mutation into a bulkier amino acid could restore the position of $\gamma \mathrm{Tol}$ to that of $\alpha \mathrm{Tol}$ in the native protein.

Parallel mutagenesis experiment showed that the A156L mutant had good affinity for $\gamma$ Tol. We therefore performed free-energy perturbation (FEP) simulations on this mutant to verify changes in the binding affinity for the two $\alpha$ Tol and $\gamma$ Tol compounds (for the technical details refer to the original publication). ${ }^{[7]}$

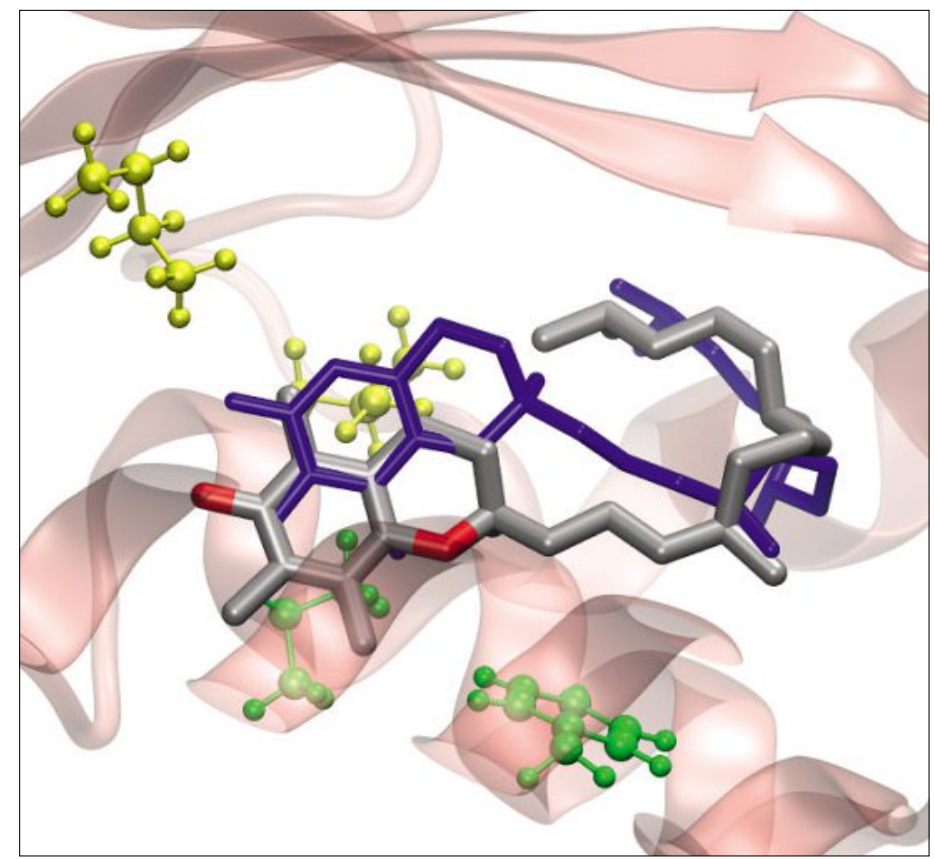

Fig. 6. Superposition of $\alpha$ Tol: $\alpha$-TTP and $\gamma$ Tol: $\alpha$-TTP structures from MD simulations. ${ }^{[7]}$ $\gamma$ Tol (blue licorice) has a different binding mode than $\alpha$ Tol (atom-code-coloured licorice). $\gamma$ Tol loses contacts with portions of the proteins (in green (PK), and interacts more tightly with other regions (yellow CPK) than $\alpha \mathrm{Tol}$. 
Indeed, we predicted that the A156L mutant would invert its selectivity, favouring binding to $\gamma$ Tol over $\alpha$ Tol. This was confirmed by differential scanning fluorimetry and competitive binding assays. ${ }^{[7]}$

\section{Mechanism of Opening/Closing of the Binding Pocket}

The opening motion of the lid is crucial for the activity of the Sec-14-like transporters. In fact, it is required for ligand exchange, and must strictly occur after binding to the specific target membrane.

MD studies on the Sec14p protein predict large motions of the mobile gate, which are assumed to be rigid-body motions. This movement is mediated and regulated by additional structural elements: a hinge module that interacts with the $\mathrm{N}$ and $\mathrm{C}$ termini of the mobile gate; and a gating module, that transduces conformational changes to the hinge module. ${ }^{[45]}$

Here, the global opening/closing motion in $\alpha$-TTP was studied by metadynamics with path collective variables. ${ }^{[46-49]}$

The MD-metadynamics run protocol followed that in ref. [47], and was performed interfacing the GROMACS program ${ }^{[50]}$ with the portable plugin for metadynamics PLUMED. ${ }^{[51]}$ Initially, the structures were relaxed following the same procedure as in ref. [7]. We guessed the transition path by linear interpolation of the coordinates of the open and closed state of the protein. We used five intermediate points along this path. Metadynamics parameters were set as $\mathrm{s}=0.3, \mathrm{Z}=0.02 \AA^{2}$ and $\lambda=7.0 \AA^{2}$. The metadynamics run took $10 \mathrm{~ns}$.

$\alpha$-TTP constitutes an optimal case study, as both the open and closed conformations of the protein are available from crystallographic analysis.

Despite the two structures suggest a simple rigid motion of the gate helix, our computational investigation reveals that the opening occurs in two steps. In the intermediate structure, the lid helix partially unfolds in its C-terminal side, exposing the opening of the cavity next to the basic-patch area. Interestingly, in this structure the loop connecting the N-terminal side of the lid to helix $\alpha 9$ is also distorted. Our simulations report global weakening of the hydrophobic interactions keeping the helix in the closed form also at the hinge region at the $\mathrm{N}$-terminal side of the lid. In particular, the side chains of F165, P200 and W163 form in the closed state a tightly packed hydrophobic cluster. At the intermediate structure, such a moiety is highly deformed. In particular, the sidechain of F165 changes orientation, accompanying a large displacement of W163 (Fig. 7). This amino acid changes its global conformation, with the dihedral angle $\chi_{1}$ passing from $\sim 178^{\circ}$ to $\sim-58^{\circ}$, consequently exposing the indole group to the solvent.

Taken into consideration that F165 is directly involved in membrane binding, ${ }^{[40]}$ our metadynamics simulations predict that the opening of the gate requires a large displacement and exposition to the solvent of hydrophobic moieties next to the protein surface. Such a process may therefore be favoured by initial adhesion to the membrane, which could screen the hinge residues from the highly polar water environment, and could help the displacement of the indole from the protein surface by inserting it to the membrane.

We finally note that the loop connecting helix $\alpha 9$ to the lid is at the protein surface in single domain Sec-14-like proteins, but it may be hindered by the presence of other domains in multi-domain proteins. This is the case of the supernatant protein factor (SPF), a multidomain protein involved in the endosynthesis of cholesterol. In this case, the Sec-14-like domain is associated to a jelly-roll C-terminal domain whose function is not well understood, but is homologous to other Golgi-dynamics domains. Binding of the two domains blocks the hinge region, avoiding any displacement similar to that observed in our simulations.

Despite several biochemical data indicating that SPF facilitates translocation of both squalene and 2,3-oxidosqualene toward the lanosterol cyclase, ${ }^{[52]}$ so far, no evidence of direct binding of either of these substrates was reported. Our simulations therefore suggest that a larger displacement of the two domains may be required to facilitate binding of the substrate in SPF.

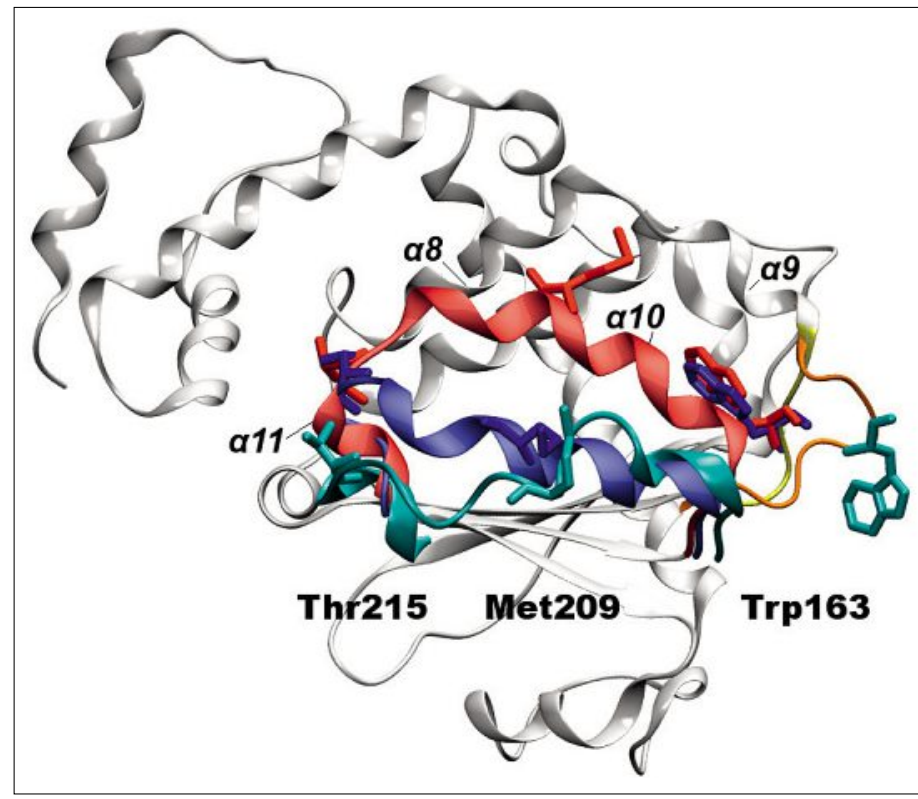

\section{Concluding Remarks}

Here, we reviewed some recent advances in our groups on vitamin transporters of the Sec-14-like family. We discovered how subtle differences in the chemical structure of substrates produce significant modification in the binding properties and in the binding geometries.

Such differences can be so deep that, in the case of CRALBP, an unexpected, new isomerase function appears when 9-cis-retinal is bound to it. On the other hand, understanding their mechanism can be the starting ground to design engineered protein variants with tailored function.

On a broader basis, molecular dynamics simulations have become a very solid and reliable method of investigation of biomolecular and biochemical phenomena. Our investigations show how today simulations and experiments can walk on each other's side, building virtuous synergies and both contributing significantly to a deeper understanding of molecular biological phenomena.

\section{Acknowledgements}

The research presented here was funded by the Swiss National Science Foundation (Grants n.: PP02_118930, PP00P2_139195 (MC), 31003A_130497 (AS)). MC acknowledges the support of the Norwegian Research Council through the $\mathrm{CoE}$ Centre for Theoretical and Computational Chemistry (CTCC) Grant Nos. 179568/V30 and 171185/V30.

Received: June 30, 2014

[1] V. A. Bankaitis, J. R. Aitken, A. E. Cleves, W. Dowhan, Nature 1990, 347, 561.

[2] A. G. Howe, C. R. McMaster, Can. J. Physiol. Pharm. 2006, 84, 29.

[3] K. E. Ile, G. Schaaf, V. A. Bankaitis, Nat. Chem. Biol. 2006, 2, 576.

Fig. 7. Superposition of crystallographic structures of $\alpha$-TTP with closed lid (red colour. PDB: 1OIZ), open lid (blue, PDB: 1OIP) and intermediate structure from metadynamics simulations. (cyan). The deformation of the loop connecting $\alpha 9$ to the lid helix in the intermediate structure, with consequent solvent exposure of W163, is evidenced by colouring the loop in orange. 
[4] A. Debant, C. Serra-Pages, K. Seipel, S. O'Brien, M. Tang, S. H. Park, M. Streuli, Proc. Natl. Acad. Sci. USA 1996, 93, 5466.

[5] R. Meier, T. Tomizaki, C. Schulze-Briese, U. Baumann,A.Stocker,J.Mol.Biol.2003,331,725.

[6] C. Panagabko, S. Morley, M. Hernandez, P. Cassolato, H. Gordon, R. Parsons, D. Manor, J. Atkinson, Biochemistry 2003, 42, 6467.

[7] R. E. Helbling, W. Aeschimann, F. Simona, A. Stocker, M. Cascella, PLoS ONE 2012, 7, e49195.

[8] C. S. Bolze, R. E. Helbling, R. L. Owen, A. R. Pearson, G. Pompidor, F. Dworkowski, M. R. Fuchs, J. Furrer, M. Golczak, K. Palczewski, M Cascella, A. Stocker, J. Am. Chem. Soc. 2014, 136, 137.

[9] M. Cascella, S. Bärfuss, A. Stocker, Arch. Biochem. Biophys. 2013, 539, 187.

[10] R. E. Helbling, C. S. Bolze, M. Golczak, K. Palczewski, A. Stocker, M. Cascella, J. Phys Chem. B 2013, 117, 10719.

[11] M. Christen, M. J. Marcaida, C. Lamprakis, J. Vaithilingam, M. Hilbert, M. Cascella, A. Stocker, submitted.

[12] J. von Lintig, P. D. Kiser, M. Golczak, K. Palczewski, Trends Biochem. Sci. 2010, 35, 400.

[13] J. W. Crabb, Y. Chen, J. T. Kapron, K. A. West, D. L. Bredberg, J. C. Saan, Invest. Ophth. Vis. Sci. 1996, 37, 3691.

[14] J. C. Saari, A. H. Bunt, S. Futterman, E. R Berman, Invest. Ophth. Vis. Sci. 1977, 16, 797.

[15] P. D. Kiser, M. Golczak, A. Maeda, K Palczewski, Biochim. Biophys. Acta 2012 $1821,137$.

[16] K. J. Palczewski, Biol. Chem. 2012, 287, 1612.

[17] J. C. Saari, M. Nawrot, B. N. Kennedy, G. G. Garwin, J. B. Hurley, J. Huang, D. E. Possin, J. W. Crabb, Neuron 2001, 29, 739.

[18] I. Golovleva, S. Bhattacharya, Z. P. Wu, N Shaw, Y. W. Yang, H. Andrabi, K. A. West, M. S. I. Burstedt, K. Forsman, G. Holmgren, O. Sandgren, N. Noy, J. Qin, J. W. Crabb, J. Biol. Chem. 2003, 278, 12397.

[19] J. C. Saari, D. L. Bredberg, N. Noy, Biochemistry 1994, 33, 3106

[20] A. Muniz, E. T. Villazana-Espinoza, B. Thackeray, A. T. C. Tsin, Biochemistry 2006 $45,12265$.

[21] J. C. Saari, J. W. Crabb, Exp. Eye Res. 2005, 81, 245 .

[22] J. C. Saari, J. Huang, D. E. Possin, R. N. Fariss, J. Leonard, G. G. Garwin, J. W. Crabb, A. H. Milam, Glia 1997, 21, 259.
[23] X. Q. He, J. Lobsiger, A. Stocker, Proc. Natl. Acad. Sci. USA 2009, 106, 18545.

[24] J. K. McBee, J. P. Van Hooser, G. F. Jang, K. Palczewski, J. Biol. Chem. 2001, 276, 48483.

[25] J. C. Saari, D. L. Bredberg, J. Biol. Chem. 1987, 262,7618

[26] M. S. I. Burstedt, O. Sandgren, G. Holmgren, K. Forsman-Semb, Invest. Ophth. Vis. Sci. 1999, $40,995$.

[27] M. S. I. Burstedt, K. Forsman-Semb, I. Golovleva, T. Janunger, L. Wachtmeister, O. Sandgren, Arch. Ophth. 2001, 119, 260.

[28] T. D. Lamb, E. N. Pugh, Progr. Retin. Eye Res. 2004, 23, 307.

[29] T. Y. Liu, E. Jenwitheesuk, D. C. Teller, R. Samudrala, Proteins 2005, 61, 412

[30] Z. P. Wu, A. Hasan, T. Y. Liu, D. C. Teller, J. W. Crabb, J. Biol. Chem. 2004, 279, 27357.

[31] A. Warshel, M. Levitt, J. Mol. Biol. 1976, 103, 227.

[32] A. Laio, J. VandeVondele, U. Rothlisberger, J. Phys. Chem. B 2002, 106, 7300.

[33] A. Laio, J. VandeVondele, U. Rothlisberger, $J$. Chem. Phys. 2002, 116, 6941.

[34] R. Rowan, A. Warshel, B. D. Sykes, M. Karplus, Biochemistry 1974, 13, 970.

[35] G. L. Catignani, Biochem. Biophys. Res. Co. 1975, 67, 66

[36] Y. Sato, K. Hagiwara, H. Arai, K. Inoue, FEBS Lett. 1991, 288, 41.

[37] M. G. Traber, H. Arai, Annu. Rev. Nutr. 1999, 19, 343.

[38] D. E. Kaempf-Rotzoll, M. Horiguchi, K. Hashiguchi, J. Aoki, H. Tamai, O. Linderkamp, H. Arai, Placenta 2003, 24, 439.

[39] M. Shichiri, N. Kono, Y. Shimanaka, M. Tanito, D. E. Rotzoll, Y. Yoshida, Y. Hagihara, H. Tamai, H. Arai, J. Biol. Chem. 2012, 287, 2926.

[40] W. X. Zhang, V. Thakur, A. Lomize, I Pogozheva, C. Panagabko, M. Cecchini, M. Baptist, S. Morley, D. Manor, J. Atkinson, J. Mol. Biol. 2011, 405, 972.

[41] K. C. Min, R. A. Kovall, W. A. Hendrickson, Proc. Natl. Acad. Sci. USA 2003, 100, 14713.

[42] R. Brigelius-Flohe, Nutr. Res. Rev. 2006, 19 174.

[43] S. E. Campbell, W. L. Stone, S. Lee, S. Whaley, H. S. Yang, M. Qui, P. Goforth, D. Sherman, D McHaffie, K. Krishnan, BMC Cancer 2006, 6, 13.

[44] A. Stocker, Ann. Ny. Acad. Sci. 2004, 1031, 44.

[45] M. M. Ryan, B. R. S. Temple, S. E. Phillips, V. A. Bankaitis, Mol. Biol. Cell 2007, 18, 1928.

[46] A. Laio, M. Parrinello, Proc. Natl. Acad. Sci. USA 2002, 99, 12562
[47] D. Branduardi, F. L. Gervasio, M. Parrinello, J. Chem. Phys. 2007, 126, 054103.

[48] A. Berteotti, A. Cavalli, D. Branduardi, F. L. Gervasio, M. Recanatini, M. Parrinello, J. Am. Chem. Soc. 2009, 131, 244.

[49] N. Besker, F. L. Gervasio, Methods Mol. Biol. 2012, 819, 501.

[50] D. Van der Spoel, E. Lindahl, B. Hess, G. Groenhof, A. E. Mark, H. J. C. Berendsen, J. Comput. Chem. 2005, 26, 1701.

[51] M. Bonomi, D. Branduardi, G. Bussi, C. Camilloni, D. Provasi, P. Raiteri, D. Donadio, F. Marinelli, F. Pietrucci, R. A. Broglia, M. Parrinello, Comput. Phys. Commun. 2009, 180 , 1961.

[52] A. Chugh, A. Ray, J. B. Gupta, Progr. Lipid Res. 2003, 42, 37 . 